\title{
Oscillation-based DFT for Second-order OTA-C Filters
}

\author{
Masood-ul-Hasan, Yichuang Sun, Xi Zhu, and James Moritz \\ School of ECEE \\ University of Hertfordshire, \\ Hatfield, Herts, AL10 9AB, UK \\ y.sun@herts.ac.uk
}

\begin{abstract}
We propose an easily implemented and low-cost design-for-testability scheme for OTA-C filters based on an oscillation-based test (OBT) methodology. The OBT method is a vectorless output test strategy easily applicable to built-in selftest. During test mode, the filter under test is converted into an oscillator by establishing the oscillation condition in its transfer function. The oscillator frequency can be measured using digital circuitry and deviations from the cut-off frequency indicate faulty behaviour of the filter. The proposed method is suitable for both catastrophic and parametric fault diagnosis and is effective in detecting single and multiple faults. The validity of the proposed method has been verified using comparison between faulty and fault-free simulation results of twointegrator loop, Tow-Thomas and KHN OTA-C filters. Simulation results for $2^{\text {nd }}$ order filters using a $0.25 \mu \mathrm{m}$ CMOS technology show that the proposed oscillation-based test strategy has more than $96 \%$ fault coverage and, with a minimum number of extra components, requires a negligible area overhead.
\end{abstract}

\section{INTRODUCTION}

In the last fifteen years or so, tremendous progress has been made in integrated analogue filter research and development, however, testability of analog integrated filters is still rather unstructured since testability is not yet a precisely defined term in the analogue world. Consumer electronic systems often require design for testability (DFT) techniques, including built-in self-test structure for analogue and mixed-signal ICs. DFT for analogue circuits is one of the most challenging tasks in mixed-signal ASIC design [1] due to the sensitivity of the circuit parameters to component variations and process technologies. Furthermore, the numbers of I/O pins of analogue ICs are relatively small compared to that of digital circuits and the complexity due to continuous signal values in the time domain and the inherent interaction between various circuit parameters make design of an efficient DFT for functional verification and diagnosis extremely difficult.

Therefore an efficient DFT procedure is required which uses a single signal as input or a self-generated input signal, has access to several internal nodes and must contain sufficient information about the circuit under test in the output [2]. The DFT of analog circuits can be generally divided into two categories; the first deals with controllability and observability of the internal nodes and the second is to convert the circuit under test (CUT) function and generate an output signal which contains the CUT performance to determine its malfunction. Oscillation-based test (OBT) procedures for analog circuits, based on transformation of the CUT into an oscillator have been recently introduced [3, 4]. The oscillation-based DFT structure uses vectorless output frequency comparison between fault-free and faulty circuits and consequently reduces test time, cost, complexity and area overhead. In this paper we present an easily implemented and low-cost DFT scheme for OTA-C filters using this oscillationbased test methodology. The proposed strategy shows greatly improved detection and diagnostic capabilities for a number of catastrophic and parametric faults. Application of the oscillation-based DFT scheme to two integrator loop, TowThomas, and KHN biquads is discussed, because these structures are commonly used as building blocks for high order filters.

The format of this paper is as follows; the quadrature OTA-C oscillator and oscillation conditions are discussed in Section 2. Section 3 introduces filter to oscillator conversion schemes. Analog fault modelling is presented in Section 4. Test simulation results are given in Section 5, and Section 6 contains conclusions.

\section{QUADRATURE OSCILLATOR}

An ideal quadrature oscillator consists of two lossless integrators (inverting and non-inverting) cascaded in a loop, resulting in a characteristic equation with a pair of roots lying on the imaginary axis of the complex frequency plane. Most of the existing theory for sinusoidal oscillator analysis models the oscillator structure with a basic feedback loop [5]. The feedback loop may be positive, negative or a combination of both. The quadrature oscillator model can ideally be described by a second-order characteristic equation as:

$$
\left(s^{2}-b s+\omega_{0}^{2}\right) V_{0}(s)=0
$$




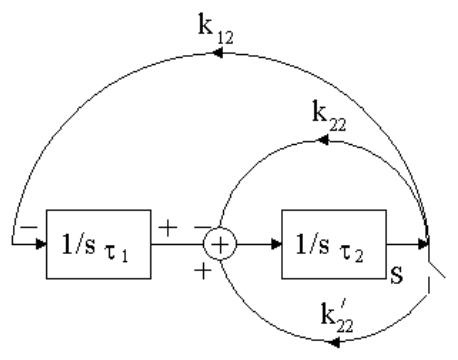

Figure 1. Converting a two-integrator loop network into an oscillator using extra feedback path $k_{22}^{\prime}$

The oscillation frequency $\omega_{0}$ can be obtained by first substituting $\mathrm{s}=\mathrm{j} \omega$ into equation (1) and considering real and imaginary parts separately. The oscillation conditions are obtained from the Barkhausen criterion [3]. Fig.1. shows a general topology for conversion of two-integrator loop network into a quadrature oscillator by establishes the oscillation condition. The pole polynomial $\mathrm{D}(\mathrm{s})$, pole angular frequency $\omega_{0}$ and quality factor $\mathrm{Q}$ of the network can be defined, respectively as:

$$
\begin{aligned}
& D(s)=\tau_{1} \tau_{2} s^{2}+k_{22} \tau_{2} s+k_{12} \\
& \omega_{o}=\sqrt{\frac{k_{12}}{\tau_{1} \tau_{2}}}, Q=\frac{1}{k_{22}} \sqrt{k_{12} \frac{\tau_{1}}{\tau_{2}}}
\end{aligned}
$$

Equation (2) shows that the network can oscillate at resonant frequency $\omega_{o}$ if it has infinite Q (i.e. if $k_{22}=0$ ). To keep the frequency of the oscillation at the un-damped pole, the quality factor should be increased only by changing the values of the components which do not affect $\omega_{0}$. It can be seen from the equations (3) that the quality factor $\mathrm{Q}$ tends to infinity if $k_{22}$ goes to zero. We proposed a feedback method to convert the two-integrator loop OTA-C filters into oscillators without affect their performance. The proposed method is based on the cancellation of $\mathrm{Q}$ factor damping coefficient $k_{22}$ by introducing an extra positive feedback path $k_{22}^{\prime}$ in filter configuration as shown in Fig.1. The proposed filter into oscillator conversion methodology requires minimum additional components and is easily incorporated at the integrator output node without producing significant effects on the performance of the filters.

\section{FILTER INTO OSCILLATOR}

Any type of OTA-C filter of second or higher order has the potential for oscillation. This ability can be used to convert the filter under test (FUT) to an oscillator by establishing the oscillation condition in its transfer function. In this section we present the implementation of proposed method based on an extra feedback topology for $2^{\text {nd }}$-order OTA-C filters. The filter into oscillator conversion method is discussed for the twointegrator loop, Tow-Thomas and KHN $2^{\text {nd }}$-order filters because they are commonly used by themselves or as basic building blocks for high-order OTA-C filters.

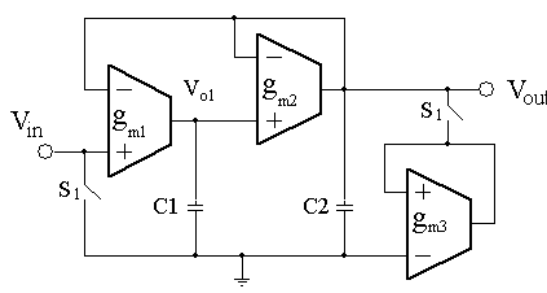

(a)

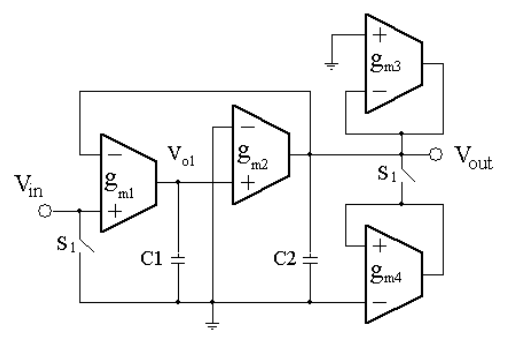

(b)

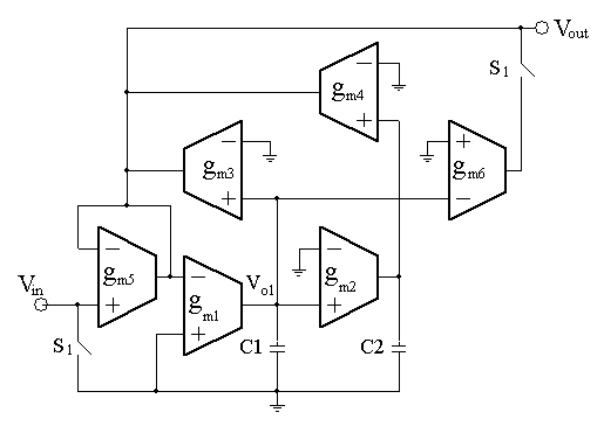

(c)

Figure 2. Testable (a) two-integrator loop,(b) Tow-Thomas and (c) KHN OTA-C filters using an extra OTA and two MOS switches

Fig. 2 shows testable integrator loop OTA-C filters using the strategy given in Fig. 1. In the normal filter mode, the switch $\mathrm{S}_{1}$ is open and the FUT operates as a second order filter. By closing $S_{1}$, the filter networks will become oscillators, due to the extra feedback path now in the circuits. The characteristic equation of the resulting oscillators can be written for the two-integrator loop, Tow-Thomas and KHN filters as (respectively):

$$
\begin{aligned}
& {\left[s^{2}+\frac{\left(g_{m 2}-g_{m 3}\right)}{C_{2}} s+\frac{g_{m 1} g_{m 2}}{C_{1} C_{2}}\right] V_{\text {out }}(s)=0} \\
& {\left[s^{2}+\frac{\left(g_{m 3}-g_{m 4}\right)}{C_{2}} s+\frac{g_{m 1} g_{m 2}}{C_{1} C_{2}}\right] V_{\text {out }}(s)=0}
\end{aligned}
$$




$$
\left[s^{2}+\frac{g_{m 1}\left(g_{m 3}-g_{m 6}\right)}{g_{m 5} C_{1}} s+\frac{g_{m 4} g_{m 1} g_{m 2}}{g_{m 5} C_{1} C_{2}}\right] V_{\text {out }}(s)=0 \text { (6) }
$$

From equations (4)-(6) we can see that the angular frequency $\omega_{0}=\sqrt{\frac{g_{m 1} g_{m 2}}{C_{1} C_{2}}}$ for the two-integrator loop (TIL) and Tow-Thomas (TT) filters and $\omega_{0}=\sqrt{\frac{g_{m_{4} g_{m 1} g_{m 2}}}{g_{m_{5} C_{1} C_{2}}}}$ for the KHN filter. The oscillation conditions for the TIL, TT and KHN filters are given by $g_{\mathrm{m} 2}=g_{\mathrm{m} 3}, g_{\mathrm{m} 3}=g_{\mathrm{m} 4}$ and $g_{\mathrm{m} 3}=g_{\mathrm{m} 6}$ respectively.

Equations (4)-(6) also show that the transconductance gains appearing in the expression for $\omega_{0}$ are different from the transconductance gain for oscillation conditions. Ideally we can tune the oscillator frequency via $\mathrm{g}_{\mathrm{m} 1}$ and $\mathrm{g}_{\mathrm{m} 2}$ for TIL and TT and via $g_{\mathrm{m} 1}, g_{\mathrm{m} 2}, g_{\mathrm{m} 4}$ and $\mathrm{g}_{\mathrm{m} 5}$ for KHN converted oscillators without affecting the amplitude of the oscillation however, when parasitics are considered, $\mathrm{g}_{\mathrm{m} 3}, \mathrm{~g}_{\mathrm{m} 4}$ and $\mathrm{g}_{\mathrm{m} 6}$ must also be tuned to maintain constant amplitude of the corresponding oscillator.

\section{FAUlT MODELLING}

In order to quantify the fault coverage of the proposed method, an accurate and realistic list of catastrophic and parametric faults is required. Catastrophic faults cause the total failure of the circuit. These types of fault are easy to detect but difficult to locate and correct. Parametric faults are caused by deviation in the process parameters and manufacturing process. Parameter deviation faults are more difficult to detect since the circuit can behave in an acceptable manner. We have considered two types of catastrophic fault; the stuck-short fault (SSF) and stuck-open fault (SOF). Several research results show that $90 \%$ of the observed hard faults consist of shorts and opens in transistors, diodes, and capacitors. All catastrophic faults are considered to be in transistors, capacitors and interconnections and each fault type is modelled for PSPICE simulation using either a low impedance of $10 \Omega$ for SSF or high impedance of $100 \mathrm{M} \Omega$ for SOF. All parametric faults are considered to be in transconductance, aspect ratio, channel width, channel length and the capacitance of the capacitors.

\section{Simulation Results}

The testable two-integrator loop, Tow-Thomas and KHN OTA-C filters in Fig.2 were simulated using PSPICE level 7 models in TSMC $0.25 \mu \mathrm{m}$ CMOS technology for verification of the proposed OBT structures. The filters were designed to have a cut-off frequency of $35 \mathrm{MHz}$ with a unity quality factor $\mathrm{Q}$, using the operational transconductance amplifier circuit shown in Fig.3. An equal transconductance design was adopted with $\mathrm{gm}=830 \mu \mathrm{S}$ and circuit capacitances $\mathrm{C} 1=$ $5.88 \mathrm{pF}$ and $\mathrm{C} 2=2.44 \mathrm{pF}$.

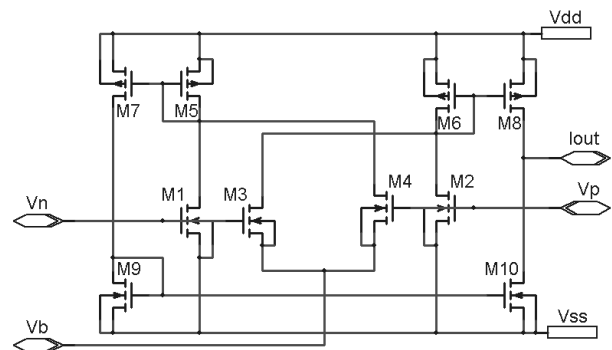

Figure 3. Transistor level schematic diagram of OTA used for simulation

The simulation results confirm that the self-starting stable oscillation of the filters is achieved by the circuit modifications described in Section 3. Simulation results demonstrate that the modified filters incorporating MOS switches and an extra OTA have similar performance to the original filters with cut-off frequency of $33 \mathrm{MHz}$. As an example, Fig.4 shows the frequency response of the TIL filter under test and time domain oscillation response of the converted corresponding oscillator. The fault-free oscillation frequencies were $30.7 \mathrm{MHz}, 30.2 \mathrm{MHz}$ and $24.5 \mathrm{MHz}$ for TIL, TT and KHN filters respectively. The oscillation frequencies are slightly shifted from the cut-off frequency of the filter due to the parasitic capacitances of the OTA and nonlinearity of the MOS switches.

To quantify the fault coverage and efficiency of the OBT strategy, a number of different faults were injected into the filters under test according to the methodology described in Section 4. To determine the undetectable tolerance band for the frequency, a Monte Carlo analysis, considering 5\% tolerance for all components was performed. The Fast Fourier Transform (FFT) of the output signal shows that the oscillation signal has lower and upper frequency deviation bounds of $-3.25 \%$ and $+3.75 \%$ respectively. The faults which produced oscillation frequency deviation outside this tolerance band were considered detectable. Tables I and II present the effects of the injected catastrophic and parametric faults on the oscillation frequencies and percentage deviations from the fault-free frequency.
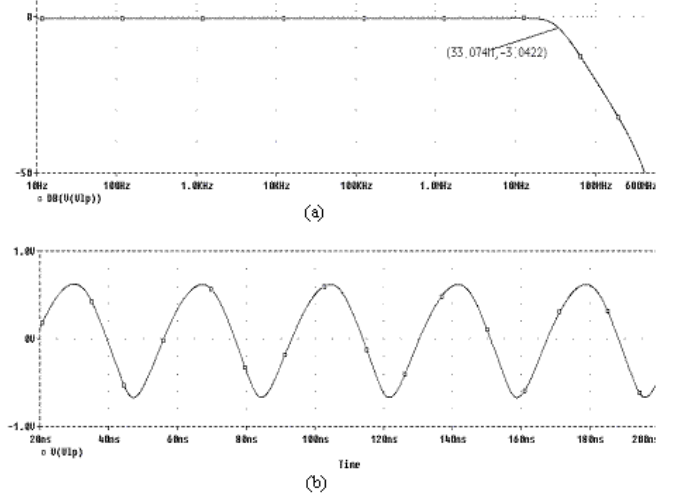

Figure 4. (a) Cut-off frequency of the filter under test and (b) oscillation frequency of the converted oscillator 
TABLE I. INJECTED CATASTROPHIC FAULTS IN THE FILTER CIRCUITS

\begin{tabular}{|c|c|c|c|c|c|c|c|c|c|c|}
\hline \multicolumn{2}{|c|}{$\begin{array}{c}\text { Faulty } \\
\text { Component }\end{array}$} & \multicolumn{3}{|c|}{ TIL } & \multicolumn{3}{|c|}{ TT } & \multicolumn{3}{|c|}{ KHN } \\
\hline $\begin{array}{c}C_{1} \\
M_{3,4}\end{array}$ & $\begin{array}{c}C_{2} \\
M_{3,4}\end{array}$ & $\begin{array}{c}f_{\text {OSC }} \\
\mathrm{Hz}\end{array}$ & $\begin{array}{c}V_{\text {OUT }} \\
V_{P P}\end{array}$ & $\begin{array}{c}\Delta f o / f o \\
\%\end{array}$ & $\begin{array}{c}f_{\text {OSC }} \\
\mathrm{Hz}\end{array}$ & $\begin{array}{c}V_{\text {OUT }} \\
V_{P P}\end{array}$ & $\begin{array}{c}\Delta f o / f o \\
\%\end{array}$ & $\begin{array}{c}\text { fosC } \\
\boldsymbol{H z}\end{array}$ & $\begin{array}{c}V_{\text {OUT }} \\
V_{P P}\end{array}$ & $\begin{array}{c}\Delta f o / f o \\
\%\end{array}$ \\
\hline $5.88 \mathrm{pF}$ & $2.44 \mathrm{pF}$ & $3.07 \mathrm{E}+07$ & 0.95 & 0 & $3.02 \mathrm{E}+07$ & 0.84 & 0 & $2.45 \mathrm{E}+07$ & 0.88 & 0 \\
\hline SOF & $2.44 \mathrm{pF}$ & $1.21 \mathrm{E}+08$ & 0.60 & 294.7 & $1.17 \mathrm{E}+08$ & 0.49 & 288.7 & $8.15 \mathrm{E}+07$ & 1.99 & 232.7 \\
\hline $5.88 \mathrm{pF}$ & SOF & $5.90 \mathrm{E}+07$ & 1.12 & 92.43 & $5.58 \mathrm{E}+07$ & 0.97 & 84.77 & $5.05 \mathrm{E}+07$ & 0.54 & 106.2 \\
\hline SOF & SOF & $1.75 \mathrm{E}+08$ & 1.66 & 471.9 & $1.86 \mathrm{E}+08$ & 1.55 & 515.9 & $1.32 \mathrm{E}+08$ & 2.33 & 438.8 \\
\hline M3s,d & SOF & $3.43 \mathrm{E}+07$ & 1.32 & -11.7 & $\mathrm{NO}$ & - & - & $1.45 \mathrm{E}+07$ & 1.48 & -40.8 \\
\hline M4s,d & SOF & $2.83 \mathrm{E}+07$ & 1.75 & 7.6 & $3.42 \mathrm{E}+07$ & 0.95 & 13.25 & $3.55 \mathrm{E}+07$ & 0.33 & 44.9 \\
\hline M3g & SOF & - & - & - & - & - & - & $1.50 \mathrm{E}+07$ & 1.46 & -38.8 \\
\hline M4g & SOF & - & - & - & - & - & - & $3.70 \mathrm{E}+07$ & 0.19 & 51.02 \\
\hline $\mathrm{All}^{*}$ & SSF & $\mathrm{NO}$ & $\mathrm{NO}$ & $\mathrm{NO}$ & $\mathrm{NO}$ & $\mathrm{NO}$ & $\mathrm{NO}$ & $\mathrm{NO}$ & $\mathrm{NO}$ & $\mathrm{NO}$ \\
\hline
\end{tabular}

Row $1=$ Fault free oscillation and amplitude, $\mathrm{SOF}=$ Stuck Open Fault, $\mathrm{SSF}=$ Stuck Short Fault, $\mathrm{NO}=$ no oscillation and $*=\mathrm{SSF}$ between any two terminals

TABLE II. INJECTED PARAMETRIC FAULTS IN THE FILTER CIRCUITS

\begin{tabular}{|c|c|c|c|c|c|c|c|c|c|}
\hline Comp & \multicolumn{3}{|c|}{$\mathrm{C}_{1}$ or $\mathrm{C}_{2}$} & \multicolumn{3}{|c|}{$\mathrm{C}_{1} \& \mathrm{C}_{2}$} & \multicolumn{3}{|c|}{$\mathbf{g}_{\mathrm{m}}$} \\
\hline \multirow{2}{*}{$\begin{array}{c}\text { \% of } \\
\text { Errors }\end{array}$} & \multicolumn{3}{|c|}{$\Delta f o / f o \%$} & \multicolumn{3}{|c|}{$\Delta f o / f o \%$} & \multicolumn{3}{|c|}{$\Delta f o / f o \%$} \\
\hline & $T I L$ & $T T$ & $K H N$ & $T I L$ & $T T$ & $K H N$ & $T I L$ & $T T$ & $K H N$ \\
\hline$-50 \%$ & 31.54 & 32.45 & 24.49 & 64.16 & 63.58 & 55.10 & -50 & -49.67 & -46.94 \\
\hline$-40 \%$ & 22.86 & 23.17 & 16.33 & 45.70 & 45.03 & 38.78 & -40.18 & -40.40 & -36.73 \\
\hline$-30 \%$ & 15.23 & 15.89 & 10.20 & 30.46 & 30.46 & 24.49 & -30.40 & -30.46 & -26.54 \\
\hline$-20 \%$ & 9.82 & 9.93 & 6.12 & 18.49 & 18.54 & 14.29 & -20.61 & -20.53 & -18.37 \\
\hline$-10 \%$ & 4.37 & 4.64 & 4.08 & 8.71 & 8.61 & 6.12 & -10.86 & -9.93 & -10.20 \\
\hline$+10 \%$ & -4.34 & -3.97 & -4.08 & -7.60 & -7.29 & -6.12 & 9.92 & 9.93 & 9.39 \\
\hline$+20 \%$ & -7.60 & -7.29 & -6.12 & -14.12 & -13.25 & -14.29 & 20.68 & 19.87 & 18.37 \\
\hline$+30 \%$ & -10.86 & -10.60 & -10.21 & -18.46 & -18.54 & -18.37 & 29.39 & 26.49 & 24.49 \\
\hline$+40 \%$ & -14.12 & -13.91 & -12.25 & -23.91 & -23.18 & -24.49 & 33.73 & 31.46 & 32.65 \\
\hline$+50 \%$ & -16.31 & -16.56 & -14.29 & -28.25 & -27.82 & -28.57 & 43.51 & $\mathrm{NO}$ & 40.82 \\
\hline
\end{tabular}

The sensitivity of frequency deviation with respect to the injected faults depends upon the relationship between the faulty components and the oscillation frequency. All the catastrophic faults and most of the parametric faults are detectable except a small number of parametric faults in the output mirror transistors. These faults can not be detected because the defective output mirror results in frequency deviation below the tolerance limits. Altogether, 97 faults were injected into the testable filters, including parametric faults with $\pm 10 \%$ to $\pm 50 \%$ deviations in parameters, as described in Section 4. Sixty-four parametric faults were detectable and most were diagnosable, except those which produced similar frequency deviations. Catastrophic faults considered were shorts and opens in the components and between interconnections. All 27 catastrophic faults were detected, but were difficult to diagnose, especially those faults that saturated the filter under test. From the above results it is clear that a high success ratio was obtained for both parametric and catastrophic fault detection. Overall fault coverage of more than $96 \%$ was obtained.

\section{CONCLUSIONS}

In this paper, we have proposed a vectorless, dynamic DFT technique for $2^{\text {nd }}$-order OTA-C filters, based on converting the filter into an oscillator using an extra OTA and two MOS switches. The OBT technique needs only measurement of the frequency deviation to detect faults, hence requires short test times and has good immunity to noise. The design is easily implemented with little area overhead and has negligible impact on filter performance. The effectiveness of the proposed OBT strategy has been demonstrated through extensive simulation of different faults in modified two-integrator loop, Tow-Thomas, and KHN OTA-C filters. Fault simulation results have shown that the proposed oscillation-based test technique provides high fault coverage of $96 \%$ and is capable of simultaneously detecting single and multiple faults. The OBT technique is suitable for BIST filter synthesis and could be easily implemented in CAD tools for filter design.

\section{REFERENCES}

[1] G. Russell and D. Learmouth, "Testing analogue functions using M-sequence”, Electron. Lett., Vol. 29, pp.1818-1819, Oct. 1993.

[2] G. Huertas, D. Vazquez, E. J. Peralias, A. Rueda, and J. L. Huertas,"Testing mixed-signal cores: practical oscillation-based test in an analog macrocell”, IEEE Design Test Comput., Vol. 9, No. 6, pp.73-82, Nov-Dec.2002.

[3] K. Arabi and B. L. Kaminska, "Oscillation-test methodology for low-cost testing of active analog filters", IEEE Trans. Instrum. Meas., Vol. 48, No. 4, pp.798-806, Aug. 1999.

[4] M. Hasan, Y. Sun and J. Moritz, "Fault detection using oscillationbased DFT for bandpass OTA-C filters" ASP Symposium, Oxford, 2006.

[5] A. Rodriguez-Vazquez, B. Linares-Barranco, J. L. Huertas and E. Sanchez-Sinencio, "On the design of voltage-controlled sinusoidal oscillators using OTAs," IEEE Trans. on Circuits and Systems, Vol.37, No.2, pp.198-211, 1990. 